\title{
Autoestima y comprensión numérica y de lectura en alumnos del cuarto grado de primaria de una institución educativa estatal
}

\author{
Self-esteem and numerical and reading comprehension in fourth grade \\ students from a state educational institution
}

\author{
Jaime Percy Guzmán Grados ${ }^{1}$ \\ Universidad Tecnológica del Perú
}

Recibido: $26-03-21$

Aceptado: 25 - $04-21$

Publicado: $18-06-21$

\section{Resumen}

Estudio de tipo sustantivo con un diseño descriptivo y correlacional, para determinar la relación entre la autoestima y la comprensión numérica y de lectura en alumnos del cuarto grado de primaria de una institución educativa estatal. La muestra estuvo conformada por 86 escolares: 38 mujeres (44.2\%) y 48 varones (55.8\%) del distrito de El Agustino en Lima Metropolitana. Se usaron tres instrumentos de medición: el Inventario de Autoestima de Coopersmith, y las Pruebas de Comprensión Numérica y de Comprensión Lectora para alumnos del Nivel Primario. Los alumnos presentan un nivel Promedio Bajo en autoestima, y un desempeño Promedio en comprensión numérica y en comprensión lectora; no se comprobó una relación entre la autoestima y la comprensión numérica en la muestra total $(\mathrm{Rho}=.142 ; p=.191)$, ni en varones $(\mathrm{Rho}=.162 ; p=.272)$ ni en mujeres $(\mathrm{Rho}=.107 ; p=.521)$. Tampoco se comprobó una relación entre la autoestima y la comprensión lectora en la muestra completa $(\mathrm{Rho}=.206$; $p=.057)$; pero sí se dan diferencias de género y en los varones la relación es clara y muy significativa $(\mathrm{Rho}=.432 ; p=.002)$, y para las mujeres es prácticamente inexistente $(\mathrm{Rho}=$ -.024; $p=.884)$. Se comprobó una relación muy significativa entre la comprensión numérica y la comprensión lectora $(\mathrm{Rho}=.427 ; p<.001)$; muy notoria en varones $(\mathrm{r}=.522 ; p<.001)$ y mujeres $(\mathrm{Rho}=.453 ; p=.004)$. Varones y mujeres no difieren en autoestima $(p=.760)$, ni en comprensión numérica $(p=.305)$ ni en comprensión lectora $(p=.093)$.

Palabras clave: autoestima; comprensión numérica; comprensión lectora; alumnos del cuarto grado de primaria; institución educativa estatal.

\begin{abstract}
A substantive-type study with a descriptive and correlational design, to determine the relationship between self-esteem and numerical and reading comprehension in students of the fourth grade of primary school of a state educational institution. The sample consisted of 86 schoolchildren: 38 women (44.2\%) and 48 men (55.8\%) from the El Agustino district in Met-
\end{abstract}

1 Docente en la Universidad Tecnológica del Perú. Lima, Perú.

E-mail: percygg23@yahoo.com ORCID: https://orcid.org/0000-0003-2731-9321

(C) Los autores. Este artículo es publicado por la Revista de Investigación en Psicología de la Facultad de Psicología, Universidad Nacional Mayor de San Marcos. Este es un artículo de acceso abierto, distribuido bajo los términos de la licencia Creative Commons Atribución 4.0 Internacional (CC BY 4.0) [https://creativecommons.org/licenses/by/4.0/deed.es] que permite el uso, distribución y reproducción en cualquier medio, siempre que la obra original sea debidamente citada de su fuente original. 
ropolitan Lima. Three measurement instruments were used: the Coopersmith Self-Esteem Inventory, the Numerical Comprehension, and the Reading Comprehension Test for the Primary Level. The students present an Average Low level in self-esteem, and an Average performance in numerical comprehension and reading comprehension; a relationship between self-esteem and numerical comprehension was not found in the total sample (Rho $=.142 ; p=$ $.191)$, neither in men $(\mathrm{Rho}=.162 ; p=.272)$ nor in women $(\mathrm{Rho}=.107 ; p=.521)$. Nor was a relationship between self-esteem and reading comprehension found in the complete sample $(\mathrm{Rho}=.206 ; p=.057)$; but there are gender differences and in men the relationship is clear and highly significant ( $\mathrm{Rho}=.432 ; p=.002)$, and for women it is practically non-existent $(\mathrm{Rho}=-.024 ; p=.884)$. A significant relationship was found between numerical comprehension and reading comprehension $(\mathrm{Rho}=.427 ; p<.001)$; very noticeable in men $(\mathrm{r}=.522 ; p<$ $.001)$ and women $(\mathrm{Rho}=.453 ; p=.004)$. Men and women do not differ in self-esteem $(p=$ $.760)$, neither in numerical comprehension ( $p=.305)$ nor in reading comprehension $(p=.093)$.

Keywords: self-esteem; numerical comprehension; reading comprehension; fourth grade students; state educational institution.

Las limitaciones educativas en nuestra patria son bastante conocidas y ocupan un lugar importante y permanente en la agenda académica, social y política de la comunidad, como una de las problemáticas en espera de encontrar alternativas viables de solución a la altura de las crecientes demandas modernas de versatilidad, dominio de la tecnología, capacidad de adaptación al cambio y eficacia en las respuestas. Sin embargo, el punto de partida para los estudiantes, junto con el desarrollo del lenguaje y la experiencia social indispensable, siempre residirá en la suficiencia en los aspectos académicos básicos ligados a la lectura, escritura, el concepto de número y las operaciones aritméticas elementales. Sobre esas bases es que se edificarán crecientes habilidades superiores potenciadas por el pensamiento concreto-funcional y luego conceptual; que, en interacción con las realizaciones escolares, familiares y sociales, se traducirán en la formación de individuos preparados para enfrentar los exigentes desafíos educativos y de rendimiento del siglo XXI (Trahtemberg, 2004, 2007).

El presente estudio se inscribe dentro del objetivo de aportar mayores conocimientos sobre las características académicas de nuestros estudiantes en formación en el nivel primario, en los dos aspectos fundamentales para su progreso en la escuela como son la comprensión de lectura y la comprensión numérica, y dentro del campo socioemocional, sobre la autoestima (AU). Este último es un constructo muy socorrido desde hace décadas y con numerosos señalamientos teóricos sobre su importancia para la vida de los alumnos, pero con escasas comprobaciones empíricas sobre su verdadera influencia. Mas en años recientes el panorama ha empezado a cambiar favorablemente (Alarcón, 2018; Chuquipoma y Fasabi, 2017; León, 2018; Luna, 2019; Meza, 2018; Palomino, 2019). 
Existen en nuestro medio investigaciones antiguas con un enfoque aislado sobre las variables que nos ocupan. Sólo en la última década es que encontramos más estudios donde se les relaciona de manera diversa. Citamos una muestra de ellos.

Delgado et al., (2005) estudiaron la comprensión lectora en alumnos del 4to al 6to grado de primaria contrastando instituciones educativas estatales y no estatales de Lima Metropolitana. Comprobaron que, aunque no siempre se cumple, sí existe una tendencia de las segundas a obtener mejores resultados que las primeras.

De Pando (2009) investigó la relación entre la comprensión lectora, las inteligencias múltiples y el rendimiento académico en alumnos de 1ro de secundaria de instituciones educativas estatales, a través del curso de ciencia, tecnología y ambiente. Encontró una relación positiva y significativa entre la comprensión lectora y la inteligencia lógico-matemática.

Ballena (2012) analizó la relación existente entre la comprensión de lectura y la resolución de problemas matemáticos en escolares del 6to grado de primaria de una institución educativa estatal. En base a una muestra de 133 estudiantes de ambos sexos encontró una relación muy significativa entre ambas variables para la muestra total y para el grupo de mujeres, mas no para los varones. Tampoco encontró diferencias en el contraste entre varones y mujeres en las dos variables señaladas.

Carranza (2015) investigó la relación entre la comprensión lectora y el rendimiento académico en matemática en estudiantes del 6to grado de primaria. Con una muestra de 141 alumnos de ambos sexos de la Región Callao, encontró una correlación moderada entre las dos variables, pero de una elevada significatividad.

Tapia (2015) examinó la relación entre la AU y el rendimiento escolar en las áreas de matemática, comunicación, personal, ciencia y ambiente, educación religiosa, educación física y arte. A partir de una muestra de 29 alumnos de ambos sexos del 5to grado de primaria de una institución educativa estatal de Chota (Cajamarca), concluyó que no existe una relación significativa entre la AU (Coopersmith) y las áreas mencionadas de rendimiento escolar, con la excepción de las áreas de matemática y de educación religiosa.

Chuquipoma y Fasabi (2017) exploraron la relación entre la AU y el rendimiento escolar, en las áreas de comunicación, lógico matemático, ciencia y ambiente y personal social. Usaron de muestra 39 alumnos de ambos sexos del 6to grado de primaria de una institución educativa estatal. Encontraron una correlación moderada entre la AU (Coopersmith) y el rendimiento escolar. No hubo diferencias significativas por género en $\mathrm{AU}$, pero sí en la variable rendimiento escolar en favor de las mujeres. 
Alarcón (2018) estudió la relación entre la AU y el rendimiento académico en el área de comunicación en alumnos del 6to grado de primaria de una institución educativa estatal. Encontró una correlación muy significativa entre ambas variables.

León (2018) investigó la relación entre la AU, la ansiedad escolar y el aprendizaje matemático en escolares del 3er grado de primaria. Concluyó que existe una correlación directa entre AU y el aprendizaje matemático, y una correlación inversa entre la ansiedad escolar y el aprendizaje matemático; en ambos casos de nivel significativo.

Meza (2018) exploró la relación entre la AU y el rendimiento académico en las áreas de matemática y de lenguaje. Con una muestra de 143 alumnos del 2 do al 6to grado de primaria de una institución educativa estatal de Huánuco, encontró una correlación baja pero con significación estadística entre la AU (Coopersmith) y el rendimiento académico en las áreas mencionadas.

Luna (2019) estudió la relación entre AU y rendimiento escolar en alumnos del 4to grado de primaria de una institución educativa estatal. En base a una muestra de 137 estudiantes de ambos sexos encontró correlaciones muy bajas entre ambas variables (Coopersmith y el registro de notas) y concluyó que no existe relación significativa entre ellas.

Palomino (2019) examinó la relación entre AU y las habilidades básicas para el aprendizaje en 22 estudiantes de ambos sexos del 4to grado de primaria de una institución educativa particular. Encontró que las habilidades matemáticas y las verbales guardaban una correlación significativa, y que los varones alcanzaban promedios más elevados que las mujeres en AU y en las habilidades verbales y matemáticas.

Dentro de los aspectos teóricos vigentes, podemos señalar que la $\mathrm{AU}$ es un constructo que cuenta con una larga historia en nuestro campo, y va de la mano con el autoconcepto que representa la descripción y el conocimiento de las propias características del individuo, algunas más centrales que otras. La AU se refiere a la valoración (implícita, intuitiva o abierta) del autoconcepto. Para algunos autores el autoconcepto es una categoría más amplia que incluye a la AU. Otros los consideran como constructos relacionados pero independientes (Guillén y Nieri, 2009; Mori, 2002).

La AU representa una realidad multidimensional referida a los muy variados campos y áreas de aplicación en una misma persona (familiar, social, laboral, académico, afectivo, etcétera). Y está sujeta a una dinámica, a una movilidad en el tiempo inevitable en el devenir del individuo a partir de sucesos concretos y de su evolución personal (González, 1999; Vargas, 2007). Igualmente, se ha señalado su importante influencia en la interacción y adaptación al medio desde una edad muy temprana, ya sea en un sentido positivo o negativo, a través de una regulación 
de la conducta como lo ha indicado Mori (2002): ofrece al individuo un sentido de continuidad en el tiempo y en el espacio, cumple un rol de integración y de organización de las experiencias significativas, regula las vivencias afectivas, y motiva y estimula conductas específicas.

La comprensión de lectura también posee una larga trayectoria como tema de estudio. Su ejercicio es complejo y supone ir bastante más allá de una mera decodificación de signos convencionales. Como lo indica Garay (2011) “...nuestro sistema cognitivo identifica las letras, realiza una transformación de letras en sonidos, construye una representación fonológica de las palabras, accede a los múltiples significados de ésta, selecciona un significado apropiado al contexto, asigna un valor sintáctico a cada palabra, construye el significado de la frase para elaborar el sentido global del texto y realiza inferencias basadas en su conocimiento del mundo."

Dentro de los modelos explicativos destaca el interactivo del procesamiento de la información de Allende, Condemarín y Milicic (1991, citado por Oré, 2012) que recogiendo la perspectiva cognitiva, señala que existe una vía doble de interacción entre el sujeto y el texto, que será ascendente en un primer momento a partir de la decodificación inicial como punto de partida; arribará a los niveles superiores de procesamiento, que suponen un enriquecimiento complejo del mensaje en función del vocabulario, redes semánticas y experiencia social del sujeto; para finalmente tomar la vía descendente de acuerdo con el significado y valoración atribuidos, su conexión con otros textos y la aplicación eventual que el sujeto realice de la experiencia. El aporte del individuo es vital en relación con el resultado final. El mismo texto, y en especial si éste es elaborado y complejo, puede llevar a diversas interpretaciones y consecuencias en diferentes lectores.

Para Van Dijik y Kinstch (1983, citado por Oré, 2012) el punto de partida es la microestructura (letras, palabras, oraciones, párrafos) que lleva al sujeto lector a construir la macroestructura (intertexto) que ya supone una elaboración cognitiva particular, una visión con significado o interpretación conjunta del texto. Ambos niveles interaccionan a partir de reglas semánticas (macrorreglas) que facilitan la síntesis y apreciación de los puntos esenciales de la lectura. Finalmente, se llega al tercer nivel denominado supraestructural, donde se relaciona y engrana la interpretación con los conocimientos aportados por otros textos, los saberes previos y con las experiencias que conforman el mundo del lector. Podemos entender a los esquemas como los conocimientos y aportes ineludibles del mismo que interactúan con el texto.

La comprensión numérica no ha sido tan estudiada en comparación con las variables precedentes. Supone el dominio paulatino de las habilidades básicas de clasificación, seriación, asimilación de símbolos matemáticos, y faenas sencillas de inducción y deducción con números. Se trata de la construcción paulatina de las 
funciones superiores características de la niñez y adolescencia que cristalizarán en la futura inteligencia lógico-matemática (Papalia, Olds y Feldman, 2005). En ese sentido, la comprensión numérica comprende específicamente identificar y diferenciar números simples y complejos, dominio de las operaciones básicas y de sus combinaciones, razonamientos de inducción y deducción con números, raciocinios por analogía de esa índole, y poder resolver problemas aritméticos sencillos (Sánchez y Reyes, 2015b).

Constituye el punto de partida para estaciones avanzadas en ese rubro como son el razonamiento numérico, que ya supone la habilidad para el cálculo, la manipulación de cifras y absolver problemas matemáticos más demandantes; y la capacidad numérica, que ya nos habla de la integración con capacidades académicas de otra índole y la asunción de niveles crecientes de pensamiento abstracto y deductivo (Sánchez y Reyes, 2015b).

Sobre la capacidad de resolución de problemas matemáticos, Nicasio (1998) puntualiza que comprende mínimamente habilidades lingüísticas (incluyendo términos y símbolos matemáticos), habilidades perceptivas, habilidades de atención y habilidades matemáticas. Deben ser acompañadas del adecuado raciocinio para comprender el problema, concebir un plan, ejecutarlo, y poder revisar y reflexionar sobre el propio desempeño (Echenique, 2006).

Dada la variedad de componentes cognitivos enumerados como sustento de las tres variables del estudio, cabría intentar una aproximación sobre las probables vías de interacción entre ellas a parir de lo señalado. Entre la comprensión lectora y la comprensión numérica aparte de los componentes cognitivos básicos de percepción, atención y memoria, comprenden en común los procesos de identificación y decodificación, representación (fonológica y simbólica), selección y atribución del significado apropiado; como puntos de entrada para la comprensión del texto y de la tarea entre manos (Garay, 2011; Oré, 2012; Papalia et al., 2005). La construcción de significados ulteriores en relación con los conocimientos previos y para delimitar una respuesta eficaz, harán uso de componentes cognitivos más elaborados como el análisis y síntesis, la inferencia, deducción y raciocinio, e, inclusive, uso de estrategias metacognitivas (Nicasio, 1998; Sánchez y Reyes, 2015b). El hallazgo usualmente repetido de una relación cercana entre la comprensión numérica y lectora (Ballena, 2012; Carranza, 2015; Pilger, Nese \& Alonzo, 2021; Vukovic \& Lesaux, 2013) tendría sustento teórico según lo señalado; sin embargo, no abundan las investigaciones que hayan estudiado en ese contexto y por separado tales componentes cognitivos. Hay hallazgos interesantes en relación con la memoria de trabajo como un factor en común significativo (Gárate et al., 2002), pero aún resta mucho campo por esclarecer al respecto. La AU es una variable que aunque cuenta con múltiples campos de aplicación en la experiencia cotidiana de toda persona, no está sujeta a la identificación de componentes cognitivos destacables más allá del autoconcepto, tomado como conocimientos acerca de uno mismo. El 
nexo de contacto y de probable influencia o interacción con las otras dos variables residiría en su naturaleza socioemocional y en su potencial como importante regulador de la conducta (Mori, 2002); la relación reportada en distintos estudios en nuestro medio entre la AU y el desempeño académico en alumnos de la escuela primaria se asentaría en esta posibilidad (Alarcón, 2018; Chuquipoma y Fasabi, 2017; León, 2018; Palomino, 2019; Tapia, 2015). Estudios extranjeros también se encaminan en esa línea (Hisken, 2011).

En síntesis de los conceptos, consideramos a la $A U$ como la valoración positiva o negativa que el sujeto hace de sí mismo, de su autoconcepto, y que se acompaña de sentimientos de valía personal y auto aceptación (Gonzáles, 1999). La comprensión de lectura es la capacidad referida a la captación del significado de los mensajes escritos, y comprende fundamentalmente la retención de lo leído, poder sistematizarlo, organizarlo y, finalmente, poder interpretarlo y valorarlo (Sánchez y Reyes, 2015a). La comprensión numérica es la base de la capacidad numérica que tiene como fundamento el dominio de la aritmética básica, y se plasma en las tareas que comprenden el uso adecuado de los números en operaciones o cálculos, razonamiento inductivo o deductivo numérico, y resolución de problemas numéricos (Sánchez y Reyes, 2015b).

El objetivo general de este estudio fue determinar la relación entre la AU y la comprensión numérica y de lectura en alumnos del cuarto grado de primaria de una institución educativa estatal; establecer el nivel de las tres variables en dicha población, las relaciones que pudieran darse entre ellas, y la influencia de la variable sexo.

\section{MÉTODO}

\section{Diseño}

Se trata de un estudio empírico con estrategia asociativa, diseño correlacional simple y aspectos comparativos (Alarcón, 2008; Ato, López-García y Benavente, 2013; Sánchez y Reyes, 1987).

\section{Participantes}

Se seleccionó por conveniencia una muestra de 86 estudiantes de cuarto grado de primaria de una institución educativa estatal del distrito de El Agustino en Lima Metropolitana, provenientes de 4 salones del turno vespertino: 38 mujeres (44.2\%) y 48 varones (55.8\%); en relación con sus edades, 74 de ellos tenían 9 años $(86 \%)$, y 12 ya alcanzaban los 10 años (14\%). El nivel socioeconómico de los participantes corresponde a las categorías $\mathrm{C}$ y D.

\section{Instrumentos}

Se usaron las siguientes pruebas psicológicas: 
a. El inventario de AU de Coopersmith (SEI), versión escolar; es una prueba diseñada para medir actitudes valorativas hacia el Sí mismo en estudiantes de 8 a 15 años (Coopersmith, 1990) y que ha sido adaptada y ampliamente utilizada en nuestro medio; explora 4 áreas a través de 58 ítems dicotómicos: Sí mismo general, Social-pares, Hogar-padres y Escuela; y posee una escala de mentiras para controlar la confiabilidad. Puede aplicarse en forma individual o colectiva y responderla suele demandar entre 20 y 30 minutos. Su calificación ofrece resultados por áreas y para el total de la prueba según cuatro niveles de AU: Baja, Promedio Bajo, Promedio Alto y Alta.

En su uso en nuestro medio con el método de confiabilidad por mitades se ha obtenido un coeficiente satisfactorio de 0.78 (Panizo, 1985; citada en Vizcarra, 1997), semejante al obtenido en otros estudios nacionales y extranjeros (Piera, 2012). Su validez de contenido se ha comprobado por el criterio de jueces (Panizo, 1985; citada en Vizcarra, 1997), y la validez de constructo por la correlación ítem-test con un nivel de significación de 0.001 (Piera, 2012).

b. Prueba de Comprensión Numérica para alumnos del Nivel Primario (PCN$N E P)$; elaborada por Sánchez y Reyes (2015b) y que está dirigida a medir la comprensión numérica en alumnos del 3er al 6to grado de primaria; su aplicación, individual o colectiva, suele durar de 45 a 60 minutos, y ha sido construida, validada y estandarizada en nuestra realidad. La prueba consta de 24 ítems con respuestas de alternativas múltiples (4), y que están relacionados con cuatro áreas: reconocimiento de números, cálculo numérico, serie numérica y problemas numéricos.

La prueba fue estandarizada en una muestra de 1,139 estudiantes de ambos sexos de los grados indicados, y provenientes de colegios estatales y particulares; cuenta con baremos diferenciados (percentiles) por grado y para colegios estatales y particulares; la confiabilidad medida con el Alfa de Cronbach es buena (0.734); y su validez fue establecida en base a 4 indicadores: el índice de discriminación de cada ítem, el análisis estadístico de cada ítem (validez interna), el índice de dificultad de cada ítem, y el índice de confiabilidad de la prueba en su conjunto y de cada ítem (Sánchez y Reyes, 2015b).

c Prueba de Comprensión Lectora para el Nivel Primario, NEP-SR (Formas A y B); también elaborada por Sánchez y Reyes (2015a) con el objetivo de medir la comprensión lectora en alumnos del 3er al 6to grado de primaria; cuenta con dos versiones equivalentes, puede aplicarse en forma individual o colectiva y su resolución suele durar de 45 a 60 minutos. Ha sido elaborada y estandarizada en nuestro medio a partir de los lineamientos del Diseño curricular nacional formulados por el Ministerio de Educación (Sánchez y Reyes, 2015a). Las dos versiones de la prueba comprenden cuatro lecturas (provenientes de textos nacionales dirigidos a estudiantes de los niveles indicados) de dificultad y de extensión 
variable y creciente; después de cada lectura aparecen cinco preguntas sobre el texto con alternativas múltiples de respuesta (4); el total de reactivos es 20.

La prueba explora los siguientes 4 aspectos: capacidad para retener lo leído, para sistematizar y organizar lo leído, para interpretar lo leído y para valorar el contenido. Su estandarización comprendió una muestra de 3,792 estudiantes de los grados señalados, de ambos sexos y provenientes de colegios estatales y particulares; cuenta con baremos (percentiles) diferenciados por grado y tipo de colegio. La confiabilidad y validez de la prueba se verificó con una muestra de 804 alumnos; el Alfa de Cronbach obtenido revela una buena confiabilidad (0.72, Forma A; 0.717, Forma B); la validez se estableció inicialmente en base al criterio de jueces, y en el análisis de los siguientes indicadores: el índice de discriminación de cada ítem, el análisis estadístico de cada ítem (validez interna), el índice de dificultad de cada ítem, y el índice de confiabilidad de la prueba en su conjunto y de cada ítem (Sánchez y Reyes, 2015a). En el presente estudio se usó la Forma B.

\section{Procedimiento y análisis de datos}

Los datos se recabaron con el empleo de las tres pruebas psicológicas indicadas, que fueron aplicadas en dos sesiones de manera colectiva y durante una misma semana, con grupos de no más de 30 alumnos por sección y durante su horario normal de clases. Para el análisis de datos se empleó el programa SPSS 24.0.

\section{RESULTADOS}

En la Tabla 1 observamos el resultado de la evaluación de las tres variables en estudio para la muestra total de 86 alumnos (38 mujeres y 48 varones, $44.2 \%$ y $55.8 \%$, respectivamente). En AU los alumnos del 4to grado de primaria obtuvieron una media que se enmarca en la categoría Promedio Bajo. En Comprensión numérica la media equivale a un percentil 50 y a una categoría Normal. En Comprensión lectora se obtuvo una media equivalente a un percentil 65 y a un resultado En la Norma.

\section{Tabla 1}

Evaluación de la Autoestima, Comprensión Numérica y Comprensión Lectora. Muestra Total

\begin{tabular}{llcc}
\hline & N & M & DE \\
\hline Autoestima & 86 & 35,56 & 6,29 \\
Comprensión numérica & 86 & 12,71 & 4,37 \\
Comprensión lectora & 86 & 12,15 & 3,35 \\
\hline
\end{tabular}

Nota. M: media, DE: desviación estándar

La evaluación de la distribución normal con la prueba de Shapiro-Wilk aplicada a las pruebas psicológicas empleadas, dio en los tres casos un resultado 
significativo (AU, $p<.001$; Comprensión numérica, $p=.029$; Comprensión lecto$\mathrm{ra}, p=.021)$ por lo que se rechaza la Ho, y podemos considerar que las distribuciones no siguen la curva normal y corresponde aplicar pruebas no paramétricas.

En la Tabla 2 observamos la relación que en la muestra total se da entre la AU y la Comprensión numérica a través del coeficiente de correlación Rho de Spearman, que es de escasa magnitud y de un nivel no significativo, y nos lleva a considerar que la relación entre ambas variables es muy limitada.

También apreciamos la relación entre la AU y la Comprensión lectora que es de tamaño moderado y de un nivel no significativo, aunque bastante cercano a serlo, y nos lleva a concluir que la relación entre ambas variables es limitada.

Asimismo, observamos la relación entre la Comprensión numérica y la Comprensión lectora que es de importante magnitud y de un nivel muy significativo, y nos lleva a considerar que la relación entre ambas variables es muy estrecha.

Para valorar los resultados según la variable sexo también fue menester evaluar previamente la distribución normal en varones y mujeres con la prueba de Shapiro-Wilk aplicada a las pruebas psicológicas empleadas $(N<50)$, y encontramos que en la evaluación de la $\mathrm{AU}$, tanto varones $(p<.001)$ como mujeres $(p=$ $.012)$ obtienen un resultado significativo $(p<.05)$ por lo que se rechaza la Ho, y podemos considerar que las distribuciones no siguen la curva normal y corresponde aplicar pruebas no paramétricas. Igual sucede con la muestra de mujeres en Comprensión lectora $(p=.040)$; y no con la muestra de varones, que sí presenta una distribución normal $(p=.804)$. Finalmente, los resultados en Comprensión numérica de ambos, varones y mujeres, indican una distribución normal ( $p=.379$ y .484, respectivamente) lo que permite la aplicación de pruebas paramétricas.

\section{Tabla 2}

Muestra total: Correlación entre las variables de estudio

\begin{tabular}{lccc}
\hline & $\mathbf{r}_{\mathbf{s}}$ & $\mathbf{p}$ & $\mathbf{N}$ \\
\hline Correlación entre la Autoestima y la Comprensión Numérica. &, 142 &, 191 \\
Correlación entre la Autoestima y la Comprensión Lectora. &, 206 &, 057 & 86 \\
Correlación entre la Comprensión Numérica y la Comprensión Lectora. &, $427^{* *}$ &, 000 \\
\hline
\end{tabular}

Nota. **. La correlación es significativa en el nivel 0,01 (bilateral), $r_{\mathrm{s}}$ : correlación Rho de Spearman

En la Tabla 3 observamos la relación en la muestra de varones entre la AU y la Comprensión numérica a través del coeficiente de correlación Rho de Spearman, que es de escasa magnitud y de un nivel no significativo, y nos lleva a considerar que en los varones la relación entre ambas variables es muy limitada. 
También apreciamos la relación entre la AU y la Comprensión lectora que es de importante magnitud y de un nivel muy significativo, y nos lleva a considerar que en los varones la relación entre ambas variables es muy estrecha.

Igualmente observamos la relación entre la Comprensión numérica y la Comprensión lectora, esta vez a través del coeficiente de correlación de Pearson, que es de importante magnitud y de un nivel muy significativo, y nos lleva a concluir que en los varones la relación entre ambas variables es muy cercana.

Tabla 3

Muestra varones: Correlación entre variables

\begin{tabular}{lccc}
\hline & $\mathbf{r}_{\mathbf{s}}$ & $\mathbf{p}$ & $\mathbf{N}$ \\
\hline Correlación entre la Autoestima y la Comprensión Numérica. &, 162 &, 272 \\
Correlación entre la Autoestima y la Comprensión Lectora. &, $432^{* *}$ &, 002 & 48 \\
Correlación entre la Comprensión Numérica y la Comprensión Lectora. &, $522^{* *}$ &, 000 \\
\hline
\end{tabular}

Nota. ${ }^{* *}$. La correlación es significativa en el nivel 0,01 (bilateral), $r_{\mathrm{s}}$ : correlación Rho de Spearman

En la Tabla 4 observamos la relación en la muestra de mujeres entre la AU y la Comprensión numérica a través del coeficiente de correlación Rho de Spearman, que es de escasa magnitud y de un nivel no significativo, y nos lleva a considerar que en las mujeres la relación entre ambas variables es muy limitada.

También apreciamos la relación entre la AU y la Comprensión lectora que es de signo negativo, de tamaño mínimo y de un nivel no significativo, y nos lleva a concluir que en las mujeres la relación entre ambas variables es inversa y prácticamente nula.

Asimismo, observamos la relación entre la Comprensión numérica y la Comprensión lectora que es de importante magnitud y de un nivel muy significativo, $\mathrm{y}$ nos lleva a considerar que en las mujeres la relación entre ambas variables es muy estrecha.

En los resultados alcanzados en las tres variables examinadas según el sexo de los participantes se aprecia una ligera superioridad de las mujeres sobre los varones en AU (medias de 35.89 y 35.29, respectivamente) y en Comprensión lectora (medias de 12.78 y 11.64, respectivamente); mientras que los varones superan ligeramente a las mujeres en Comprensión numérica (13.12 y 12.18, respectivamente); diferencias que se examinan en detalle en la Tabla 5. También se encuentra que en las tres variables los varones obtienen desviaciones estándar más elevadas que las mujeres, indicativas de una mayor dispersión de sus puntajes (AU, 7.08 y 5.20, respectivamente; Comprensión numérica, 4.96 y 3.47, respectivamente; Comprensión lectora, 3.40 y 3.21 , respectivamente). 
Tabla 4

Muestra mujeres: Correlación entre variables

\begin{tabular}{lccc}
\hline & $\mathbf{r}_{\mathrm{s}}$ & $\mathbf{p}$ & $\mathbf{N}$ \\
\hline Correlación entre la Autoestima y la Comprensión Numérica. &, 107 &, 521 \\
Correlación entre la Autoestima y la Comprensión Lectora. &,- 024 &, 884 & 38 \\
Correlación entre la Comprensión Numérica y la Comprensión Lectora. &, $453^{* *}$ &, 004 \\
\hline
\end{tabular}

Nota. **. La correlación es significativa en el nivel 0,01 (bilateral), $r_{\mathrm{s}}$ : correlación Rho de Spearman

En la Tabla 5 encontramos los resultados de la comparación entre varones y mujeres de su desempeño en las pruebas de AU y de Comprensión lectora, y observamos que en ninguno de los dos casos las diferencias llegan a ser significativas ( $p=.760$ y $p=.093$; respectivamente); aunque en Comprensión de lectura el resultado en favor de las mujeres no se aleja demasiado de dicho punto.

También observamos los resultados de la comparación entre varones y mujeres de su desempeño en la prueba de Comprensión numérica, y apreciamos que, cuando no se asumen varianzas iguales (que es el caso), la $t$ encontrada (1.031) no es significativa ( $p=.305$ ); de manera que la leve diferencia en los puntajes medios en favor de los varones, no podemos considerarla significativa.

\section{Tabla 5}

Comparación de los resultados en Autoestima y en Comprensión Lectora entre varones y mujeres utilizando la prueba $U$ de Mann-Whitney

\begin{tabular}{|c|c|c|c|c|c|c|c|}
\hline & SEXO & $\mathbf{N}$ & $\begin{array}{c}\text { Rango } \\
\text { promedio }\end{array}$ & $\begin{array}{c}\text { Suma de } \\
\text { rangos }\end{array}$ & $\begin{array}{c}\text { U de Mann- } \\
\text { Whitney }\end{array}$ & $\mathbf{Z}$ & $\mathbf{p}$ \\
\hline \multirow{3}{*}{ Autoestima } & Varones & 48 & 44,23 & 2123,00 & \multirow{2}{*}{877000} & \multirow{2}{*}{,- 305} & \multirow{2}{*}{, 760} \\
\hline & Mujeres & 38 & 42,58 & 1618,00 & & & \\
\hline & Total & 86 & & & \multirow{4}{*}{719500} & \multirow{4}{*}{-1681} & \multirow{4}{*}{,093 } \\
\hline \multirow{3}{*}{$\begin{array}{l}\text { Comprensión } \\
\text { lectora }\end{array}$} & Varones & 48 & 39,49 & 1895,50 & & & \\
\hline & Mujeres & 38 & 48,57 & 1845,50 & & & \\
\hline & Total & 86 & & & & & \\
\hline
\end{tabular}

Comparación de los resultados en Comprensión Numérica entre varones y mujeres utilizando la prueba $T$

\begin{tabular}{llllllll}
\hline & $\mathrm{F}$ & Sig. & $\mathrm{t}$ & $\mathrm{gl}$ & Sig. (bilateral) & IC 95\% \\
\hline & $\begin{array}{l}\text { Se asumen varianzas } \\
\text { Comprensión }\end{array}$ & 4,373 &, 040 &, 991 & 84 &, 325 & {$[-, 95-28]$} \\
$\begin{array}{l}\text { iguales } \\
\text { numérica }\end{array}$ & $\begin{array}{l}\text { No se asumen } \\
\text { varianzas iguales }\end{array}$ & & & 1,031 & 82,874 &, 305 & {$[-, 87-28]$} \\
\hline
\end{tabular}

\section{DISCUSIONES}

Nuestro objetivo referido a establecer el nivel de AU, de comprensión numérica y de comprensión lectora en los alumnos del 4to grado de primaria de un centro estatal, encuentra respuesta en la Tabla 1, donde apreciamos que el desempeño en 
comprensión numérica y lectora es de categoría Promedio, enmarcándose en consecuencia dentro de lo esperado. No es ese el caso para la variable AU, donde el resultado se inscribe en la categoría Promedio Bajo, lo que nos señala limitaciones en esta importante área en estos escolares, siendo difícil precisar la fuente y los alcances que pudiera llegar a tener esta condición. Estudios previos han identificado la asociación entre la AU y la condición social de los escolares que, aunque controvertida, tiende a ser directa (Milicic y Gorostegui, 1993; Tabernero, Serrano y Mérida, 2017), de manera que es razonable suponer que al menos parte de la explicación de este hallazgo resida en que la muestra investigada proviene de los niveles socioeconómicos $\mathrm{C}$ y $\mathrm{D}$.

La relación entre la AU y la comprensión numérica en los escolares bajo estudio queda establecida en la Tabla 2, donde observamos que la correlación encontrada es de escasa magnitud $\left(r_{\mathrm{s}}=.142\right)$, indicando que tal relación no alcanza a ser significativa ( $p=.191)$; y cuando investigamos en función de la variable sexo el nivel de dicha relación (Tablas 3 y 4), encontramos un resultado semejante ya que ni en los varones $\left(r_{\mathrm{s}}=.162 ; p=.272\right)$ ni en las mujeres $\left(r_{\mathrm{s}}=.107 ; p=.521\right)$ esta llega a ser significativa; por lo que podemos concluir que en nuestra muestra la AU presenta una conexión muy pobre con la comprensión numérica. Este hallazgo discrepa de lo encontrado por varios estudios en nuestro medio (León, 2018; Meza, 2018; Palomino, 2019; Tapia, 2015), pero es concordante con otros (p.ej., Luna, 2019), aunque debemos señalar que en todos estos antecedentes se evaluó la relación de la AU con el rendimiento académico en matemática y no específicamente con la comprensión numérica. En cualquier caso, se trata de un tema que merece una mayor exploración para precisar las condiciones y factores que pueden estar ejerciendo una influencia en el resultado.

La relación entre la AU y la comprensión lectora en los alumnos examinados aparece en la Tabla 2, donde apreciamos una correlación moderada $\left(r_{\mathrm{s}}=.206\right)$ que aunque no alcanza a ser significativa $(p=.057)$, merece ser relievada por su cercanía a serlo; y la exploración de esta relación tomando en cuenta la variable sexo ofrece detalles esclarecedores, porque observamos que hay una gran diferencia entre varones y mujeres (Tablas 3 y 4), ya que entre los primeros sí encontramos una fuerte relación entre la AU y la comprensión lectora $\left(r_{\mathrm{s}}=.432 ; p=.002\right)$, lo que está muy lejos de suceder en el caso de las mujeres donde la relación es prácticamente nula $\left(r_{\mathrm{s}}=-.024 ; p=.884\right)$. Asimismo, que los varones presenten una distribución normal de sus puntajes en comprensión lectora a diferencia de las mujeres, y que también sean algo más dispersos como lo indican las desviaciones estándar de unos y otras, son elementos que también contribuyen al resultado. Los estudios previos con escolares en nuestro medio han indagado la relación de la AU con el rendimiento académico en el curso de comunicación, y no específicamente con la comprensión lectora, aunque son áreas bastante relacionadas; en esa línea, encontramos resultados que sí respaldan una relación importante (Alarcón, 2018; Meza, 
2018; Palomino, 2019), pero también otros que señalan lo contario (Luna, 2019; Tapia, 2015), sin que se reporten diferencias de género como las que aparecen en nuestros datos.

En otras realidades sociales como es el caso de los Estados Unidos, Hisken (2011) luego de una revisión de diferentes fuentes, ha reportado resultados significativos entre ambas variables, señalando que el éxito en la comprensión lectora es el que alimenta la $\mathrm{AU}$, y que el fortalecimiento de esta redundará en un mejor desempeño académico.

La relación entre la comprensión numérica y la comprensión lectora en los escolares estudiados se observa en la Tabla 2, que nos muestra una correlación de importante magnitud $\left(r_{\mathrm{s}}=.427\right)$ y muy significativa $(p<.001)$ entre las dos variables; este hallazgo es ampliamente reforzado por los resultados que también obtenemos cuando examinamos lo sucedido según la variable sexo (Tablas 3 y 4), ya que tanto en los varones $\left(r_{\mathrm{s}}=.522 ; p<.001\right)$ como en las mujeres $\left(r_{\mathrm{s}}=.453\right.$; $p=.004)$ encontramos una relación muy significativa. En consecuencia, podemos estar bastante seguros acerca del importante y estrecho nexo que existe entre la comprensión numérica y la comprensión lectora en los alumnos de primaria examinados. La revisión de los escasos estudios semejantes en nuestro medio ofrece resultados que marchan en el mismo sentido, aunque no usaron como variable la comprensión numérica sino el rendimiento académico en el curso de matemáticas o la resolución de problemas de esa índole (Ballena, 2012; Carranza, 2015).

En estudios en países de habla inglesa con escolares de escuela primaria (por ejemplo, Pilger et al, 2021; Vukovic \& Lesaux, 2013), también se ha puesto de manifiesto la importancia de las habilidades en la lectura para la resolución de problemas aritméticos.

Podríamos aducir que ambos aspectos interaccionan recíprocamente para su desarrollo, estimulados por el ambiente académico, la enseñanza explícita, las tareas y las experiencias directas de realización de los niños en su medio familiar y social, para ir conformando habilidades básicas de aprendizaje que luego serán el sustento de competencias académicas avanzadas.

La influencia de la variable sexo sobre la AU y la comprensión numérica y lectora, y sobre las relaciones entre estas, ya ha sido en parte absuelto en los párrafos precedentes al señalar las semejanzas y diferencias en las correlaciones encontradas en varones y mujeres. Resta examinar y contrastar los resultados de ambos en la evaluación de las tres variables del presente estudio. La Tabla 5 nos permite comparar su desempeño tomando en cuenta los promedios y desviaciones estándar obtenidos por ambos. En AU se aprecia con claridad que no existen diferencias significativas entre unos y otras ( $p=.760)$, y lo mismo sucede en comprensión numérica ( $p=.305)$ y en comprensión lectora ( $p=.093)$, aunque en estas 
dos últimas áreas la ligera superioridad de los varones en comprensión numérica $\mathrm{y}$, en especial, de las mujeres en comprensión lectora, ya parecen configurar una tendencia a pesar de no alcanzar los niveles críticos.

En diversos estudios sobre escolares se ha reportado que no existen diferencias de género en relación con la AU (Chuquipoma y Fasabi, 2017), la comprensión de lectura (Ballena, 2012; Carrillo, 1989; Delgado et al., 2005; Díaz, 2008) y las habilidades matemáticas (Ballena, 2012). Pero también se han registrado hallazgos en el sentido contrario, señalándose una superioridad de los varones en AU (Palomino, 2019) y, sobre todo, en habilidades matemáticas (Matarazzo, 1976; Palomino, 2019; Sánchez y Reyes, 2015b); y la primacía de las mujeres en comprensión lectora (Delgado et al., 2005; Sánchez y Reyes, 2015a). Igualmente, encontrar una mayor dispersión de los puntajes de los varones en comparación con las mujeres, revelada por el mayor tamaño de las desviaciones estándar de los primeros, también se inscribe dentro de lo esperado teóricamente. La diversidad de circunstancias, de instrumentos de evaluación, y del diseño y tipo de muestras de escolares de estos estudios, probablemente influyen en la variedad de resultados e impiden que se pueda alcanzar una conclusión más sólida sobre las diferencias de género. Por el momento, las tendencias detectadas que indican un desempeño semejante en AU, una ligera superioridad de los varones en comprensión numérica y de las mujeres en comprensión lectora, son las que vendrían obteniendo un mayor respaldo.

Un tema adicional que merece ser considerado es la posibilidad a futuro de emplear los aportes de la estadística bayesiana en el análisis de los resultados, en particular por tratarse de un enfoque alternativo al clásico (frecuentista), particularmente útil cuando el tamaño de las muestras estudiadas es pequeño (como en el presente estudio) y así aminorar el riesgo de falsos positivos (error tipo I). Su empleo refuerza las inferencias estadísticas de asociación o de diferencia, que suelen ser el objetivo principal de las investigaciones, y permite una mayor certeza sobre las hipótesis en juego, ya que también toma en cuenta los conocimientos aportados por investigaciones previas (Ramos-Vera, 2020, 2021; Rendón-Macías et al., 2018). El análisis bayesiano supone una definición a priori de los resultados y luego del contraste a posteriori con estos, se tendrá una mayor o menor verosimilitud medida en la aceptación o rechazo de las hipótesis nula y alternativa consideradas. El interés por el enfoque bayesiano ha crecido en años recientes, pero su aplicación precisa de un conocimiento y preparación especializados que no suelen ser comunes.

Aun cuando las características del diseño y el tipo de muestreo empleado no permiten generalizar los resultados del estudio a otras poblaciones de escolares de primaria, podemos establecer las siguientes conclusiones: 
1. Los escolares examinados del cuarto grado de primaria de una institución educativa estatal evidencian un nivel Promedio Bajo en AU, y un desempeño Promedio en comprensión numérica y en comprensión lectora.

2. No se ha comprobado una relación entre la AU y la comprensión numérica ya que la correlación entre ambas no es significativa $\left(r_{\mathrm{s}}=.142 ; p=\right.$ .191); y dicha conclusión también es válida para los varones $\left(r_{\mathrm{s}}=.162 ; p=\right.$ .272) y las mujeres $\left(r_{\mathrm{s}}=.107 ; p=.521\right)$ de la muestra.

3. No se ha comprobado una relación entre la AU y la comprensión lectora, a pesar de presentar una correlación moderada $\left(r_{\mathrm{s}}=.206\right)$ que no alcanza por poco a ser significativa $(p=.057)$; sin embargo, sí se dan diferencias de género ya que en los varones es clara y muy significativa la relación entre estas variables $\left(r_{\mathrm{s}}=.432 ; p=.002\right)$; en cambio, para las mujeres la relación es prácticamente inexistente $(\mathrm{Rho}=-.024 ; p=.884)$.

4. Se ha comprobado una relación muy significativa entre la comprensión numérica y la comprensión lectora $\left(r_{\mathrm{s}}=.427 ; p<.001\right)$; conclusión que es reforzada por los resultados en igual sentido entre los varones $r_{\mathrm{s}}=.522$; $p<.001)$ y en las mujeres $\left(r_{\mathrm{s}}=.453 ; p=.004\right)$.

5. Los varones tienden a una mayor dispersión en sus puntajes que las mujeres en las tres variables, pero no presentan diferencias en sus resultados globales en $\mathrm{AU}(\mathrm{p}=.760)$, ni en su desempeño en comprensión numérica y lectora; ya que la ligera superioridad de los varones en comprensión numérica y, en especial, de las mujeres en comprensión lectora, no alcanzan a ser significativas ( $p=.305 ; p=.093$; respectivamente).

6. Es importante seguir realizando estudios con escolares sobre variables académicas y socioemocionales específicas en nuestro medio, que nos permitan dilucidar resultados discrepantes, seleccionar métodos e instrumentos de evaluación y las mejores condiciones de enseñanza y estímulo, para llegar a crear planes de intervención focalizados en la potenciación de sus competencias y en la eventual remediación con quienes lo precisen.

\section{REFERENCIAS}

Alarcón, P. (2018). Autoestima y rendimiento académico del área de comunicación en los estudiantes del sexto de primaria del colegio $N^{\circ} 1073$ Hermann Buse de la Guerra del distrito de Pueblo Libre (tesis doctoral). Universidad Nacional de Educación Enrique Guzmán y Valle, Lima. Extraído desde http://repositorio.une.edu.pe/handle/ UNE/3221 
Alarcón, R. (2008). Métodos y Diseños de Investigación del Comportamiento. Lima: Universidad Ricardo Palma/Editorial Universitaria.

Ato, M., López-García, J., y Benavente, A. (2013). Un sistema de clasificación de los diseños de investigación en psicología. Anales de Psicología / Annals of Psychology, 29(3), 1038-1059. https://doi.org/10.6018/analesps.29.3.178511

Ballena, R. (2012). Comprensión de Lectura y Resolución de Problemas Matemáticos en Alumnos del Sexto Grado de Primaria de una Institución Educativa Estatal del Distrito de Villa el Salvador (tesis de maestría). Universidad Ricardo Palma, Lima.

Carranza, B. (2015). Relación entre comprensión lectora y rendimiento académico de matemática en alumnos del sexto grado de primaria de una Institución Educativa de la Región Callao (tesis de maestría). Universidad San Ignacio de Loyola, Lima. Extraído desde http://repositorio.usil.edu.pe/handle/USIL/2970

Carrillo, S. (1989). Relación entre los errores en la lectura oral y la comprensión lectora en niños de 4to, 5to y 6to grado de educación primaria de sexos masculino y femenino (tesis de pregrado). Universidad Ricardo Palma, Lima.

Chuquipoma, A. y Fasabi, L. (2017). Autoestima influye en el rendimiento escolar de los alumnos del $6^{\circ}$ grado de primario de la I.E 1228 Leoncio Prado Gutiérrez del distrito de Ate-Vitarte del 2016 (tesis de pregrado). Universidad Privada Telesup, Lima. Extraído desde https://repositorio.utelesup.edu.pe/handle/UTELESUP/553

Delgado, A., Escurra, L., Atalaya, M., Álvarez, C., Pequeña, J., Santivañez, R. (2005). Comparación de la comprensión lectora en alumnos de 4to a 6to grado de primaria de centros educativos estatales y no estatales de Lima Metropolitana. Revista de Investigación en Psicología, 8 (1), 51-85.

De Pando, L. (2009). Comprensión lectora, inteligencias múltiples y rendimiento académico en alumnos del primer año de secundaria en el área de ciencia, tecnología y ambiente en los centros educativos estatales de Lima (tesis de maestría). Universidad Ricardo Palma, Lima.

Díaz, N. (2008). Relación entre la comprensión lectora y la ortografía en los alumnos del tercer grado de educación primaria de San Juan de Miraflores (tesis de maestría). Universidad Ricardo Palma, Lima.

Echenique, I. (2006). Matemáticas: Resolución de problemas. Navarra. Departamento de Educación del Gobierno de Navarra. Extraído desde http://dpto.educacion.navarra. es/publicaciones/pdf/matematicas.pdf

Gárate, M., Gutiérrez, F., Elosúa de Juan, M., Luque, J. y García, J. (2002). Memoria operativa y comprensión lectora: algunas cuestiones básicas. Revista de Acción Psicológica, 1(1), 45-68. Extraído desde http://e-spacio.uned.es/fez/view/ bibliuned:AccionPsicologica2002-numero1-0004

Garay, M. (2011). El Programa constructivista CL 1 y el incremento del nivel de comprensión lectora de los alumnos de primero de secundaria (tesis de maestría). Universidad Nacional Mayor de San Marcos, Lima. 
González Martínez, M. (1999). Algo sobre la autoestima. Qué es y cómo se expresa. Aula, 11. Extraído desde http://revistas.usal.es/ revistas_trabajo/index.php/0214-3402/article/view/3530/3550

Guillén, F. y Nieri, D. (2009). Autoestima y competitividad en una selección juvenil peruana de surf. Revista Iberoamericana de Psicología del Ejercicio y el Deporte, 4 (2), 253-269.

Hisken, L. (2011). The correlation between self-esteem and student reading ability, reading level, and academic achievement (master's degree). University of Central Missouri, USA. Extraído desde https:/d1wqtxts1xzle7.cloudfront.net/45981665/ LHisken_LibraryScience.pdf?1464323905=\&response-content-

León, D. (2018). Ansiedad escolar, Autoestima y aprendizaje matemático en estudiantes de primaria RED N 01 UGEL 02-Rímac 2016 (tesis doctoral). Universidad César Vallejo, Lima. Extraído desde https://hdl.handle.net/20.500.12692/22445

Luna, M. (2019). Autoestima y rendimiento escolar en estudiantes del 4to grado del nivel primario de la institución educativa pública "Fe y Alegría $N^{o} 17$ " de Villa el Salvador, 2018 (tesis de maestría). Universidad César Vallejo, Lima. Extraído desde https://hdl.handle.net/20.500.12692/32162

Matarazzo, J. (1976). Wechsler, Medida y valoración de la inteligencia del adulto. Barcelona: Salvat Editores.

Meza, M. (2018). Autoestima y Rendimiento Académico en niños de una Institución Educativa Estatal del distrito de Pillco Marca - Huánuco, 2016 (tesis de maestría). Universidad de Huánuco, Huánuco. Extraído desde http://repositorio.udh.edu. pe/123456789/874

Milicic, N. y Gorostegui, M. (1993). Género y autoestima: Un análisis de las diferencias por sexo en una muestra de estudiantes de Educación General Básica. Psykhe, 2 (1), 69-80.

Mori, P. (2002). Personalidad, autoconcepto y percepción del compromiso parental: sus relaciones con el rendimiento académico en alumnos del sexto grado (tesis doctoral). Universidad Nacional Mayor de San Marcos, Lima.

Nicasio, J. (1998). Dificultades de aprendizaje de las matemáticas. Madrid: Narcea.

Oré, R. (2012). Comprensión lectora, hábitos de estudio y rendimiento académico en estudiantes de primer año de una universidad privada de Lima Metropolitana (tesis de maestría). Universidad Nacional Mayor de San Marcos, Lima.

Palomino, N. (2019). Autoestima y habilidades básicas para el aprendizaje en los estudiantes de educación primaria (tesis de pregrado). Universidad de Ciencias y Humanidades, Lima.

Papalia, D., Olds, S. y Feldman, R. (2005). Psicología del Desarrollo. 9na Ed. México: McGraw-Hill.

Piera, N. (2012). Autoestima según género en estudiantes del quinto grado de la Red Educativa $N^{\circ} 4$-Ventanilla (tesis de maestría). Universidad San Ignacio de Loyola, Lima. 
Pilger, M., Nese, J. \& Alonzo, J. (2021). Parallel reading and mathematics growth for English learners: Does timing of reclassification matter? Journal of School Psychology, 85, 94-112. https://doi.org/10.1016/j.jsp.2021.02.003

Ramos-Vera, C. (2020). La alternativa metodológica del factor Bayes en la investigación clínica de nutrición. Revista Española de Nutrición Humana y Dietética, 24(4). doi: 10.14306/renhyd.24.4.1231 [ahead of print]

Ramos-Vera, C. (2021). Un caso del factor Bayes en un estudio comparativo según género del miedo a la COVID-19 en Cuba. Revista Cubana de Medicina Militar, 50(2). Extraído desde http://revmedmilitar.sld.cu/index.php/mil/article/view/980

Rendón-Macías, M., Riojas-Garza, A., Contreras-Estrada, D. y Martínez-Ezquerro, J. (2018). Análisis bayesiano. Conceptos básicos y prácticos para su interpretación y uso. Revista alergia México, 65(3), 285-298. https://doi.org/10.29262/ram.v65i3.512

Sánchez, H. y Reyes, C. (1987). Metodología y Diseños en la Investigación Científica. Lima: s.ed.

Sánchez, H. y Reyes, C. (2015a). Prueba de Comprensión Lectora para el Nivel Primario, NEP-SR, (Formas A y B). Lima: Business Support Aneth S.R.L. Universidad Ricardo Palma, Facultad de Psicología.

Sánchez, H. y Reyes, C. (2015b). Elaboración y Validación de una Prueba de Comprensión Numérica para Alumnos del Nivel Primario (PCN-NEP) de Instituciones Educativas de Santiago de Surco (UGEL 07 Lima). Lima. Universidad Ricardo Palma, Facultad de Psicología, Centro de Investigación.

Tapia, W. (2015). Autoestima y rendimiento escolar de los estudiantes de la I.E 10384 Chota 2015 (tesis de pregrado). Universidad Nacional de Cajamarca, Cajamarca. Extraído desde http://repositorio.unc.edu.pe/handle/UNC/946

Tabernero, C., Serrano, A. y Mérida, R. (2017). Estudio comparativo de la autoestima en escolares de diferente nivel socioeconómico. Psicología Educativa, 23 (1), 9-17.

Trahtemberg, L. (2004). La educación peruana en emergencia. Lima: Bruño.

Trahtemberg, L. (2007). La educación peruana frente a los retos de la globalización. Lima: Bruño.

Vargas, J. (2007). Juicio moral y autoestima en estudiantes escolares del quinto año de secundaria de colegios de Lima metropolitana (tesis de maestría). Universidad Nacional Mayor de San Marcos, Lima.

Vizcarra, H. (1997). Niveles de autoestima en universitarios que solicitan orientación vocacional en la Universidad San Martín de Porres (tesis de pregrado). Universidad Nacional Mayor de San Marcos. Lima.

Vukovic, R. \& Lesaux, N. (2013). The relationship between linguistic skills and arithmetic knowledge. Learning and Individual Differences, 23, 87-91. https://doi.org/10.1016/j. lindif.2012.10.007 
